Z. klin. Chem. u. klin. Biochem.

7. Jg., S. 155-157, März 1969

\title{
Isoenzymfraktionen der Fructose-Phosphat-Aldolase in den Blutzellen gesunder Menschen
}

\author{
Isoenzyme der Fructose-Pbosphat-Aldolase,IV. Mitteilung ${ }^{1}$ )
}

Von A. L. Dikow und V. G. Genowa

Aus den Biochemiscben Abteilungen der Wissenscbaftlicben Forscbungsinstitute für Onkologie (Direktor: Prof. N. Antscben') und fïr Hämatologie und Bluttransfusion (Direktor: Prof. V. Serafimon'), Sofia, Bulgarien

(Eingegangen am 30. Oktober 1968)

In vorliegender Arbeit werden die Isoenzymmuster der Blutzellenaldolase an Hand elektrophoretischer Trennung und ansehlic $3 e n d e m$ Nachweis der Isoenzymaktivität gegenüber Fructose-1-phosphat und Fructose-1,6-diphosphat untersucht. Die Erythrocyten, dic I.cuko-

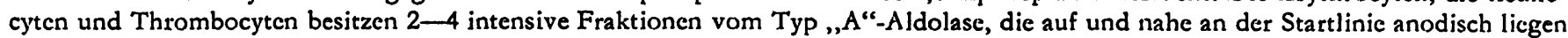
und drei weniger intensive Isoenzymfraktionen vom Typ der Aldolasc-,„C“, die sich auf der anodischen Scite des Isoenzymogramms befinden.

\section{The isoenzyme fractions of fructose phosphate aldolase in the blood cells of normal humans}

The pattern of blood cell aldolose was determined by electrophoretic scparation, followed by the detection of isoenzyme activity towards fructose-1-phosphate and fructose-1,6-diphosphate. The erythrocytcs, leucocytes and thrombocytes possess 2-4 intense fractions of type " $A$ " aldolase that lie on and near the starting line on the anode side, and thrce less intensive isocnzyme fractions of type " $C$ " aldolase that lic on the anodic side of the isoenzymogram.

Die Blutzellen (Erythrocyten, Leukocyten und Thrombocyten) besitzen einen Eigenstoffwechsel und enthalten die vollständige Enzymkette der Glykolyse. Manche Verfasser untersuchten eingehend die einzelnen Enzyme dieser Kette, einschließlich der Aldolase (EC 4.1.2.13). Die Gesamt-Aldolaseaktivität der Erythrocyten wurde hauptsächlich von 1. c. $(1,2,3,4)$ untersucht, l. c. $(5,6)$ versuchten, das Enzym auf chromatographischem Wege aufzutrennen. Die Leukocytenaldolase wurde von 1 . c. $(1,7,8,9)$, die Thrombocytenaldolase von 1 . c. $(1,10,11,12)$ untersucht. Während manche Isoenzymmuster der Blutzellen bereits erforscht wurden (Lactatdehydrogenase (EC 1.1.1.27), Glucose-6-phosphatdehydrogenase (EC 1.1.1.49), Malatdehydrogenase (EC 1.1.1.37), saure und alkalische Phosphatase (EC 3.1.3.1, 3.1.3.2), Diaphorase (EC 1.6.4.3) usw.), liegen ähnliche Untersuchungen der Aldolase noch nicht vor. Deshalb stellten wir uns die Aufgabe, im Rahmen unserer Untersuchungen über die Isoenzymfraktionen der Aldolase beim Menschen, auch die der Blutzellen bei gesunden Probanden zu erforschen.

\section{Material und Methoden \\ Bezugsquellen}

Nitro-Blau-Tetrazoliumchlorid, Calbiochem, USA; DEAE-Cellulose, Amido-schwarz 10B Fa. Schúchardt, München, Fructosc-1phosphat- $\mathrm{Na}_{2} \mathrm{salz}, \mathrm{Fa}$. Bochringer, Mannheim. Dic übrigen Bezugsquellen waren dieselben, wie in 1. c. (13).

\section{Versucbsmaterial}

Von 10 gesunden Spendern wurden unter Anwendung der Silikontechnik je $250 \mathrm{~m} /$ Blut mit einem Stabilisator (3,8proz. Lösung

1) III. Mitteilung siche Dikow, A. und M. Romanow, Zschr. inn. Med. Leipzig 23, 471 (1968). von Natrium citricum) entnommen. Dic Thrombocyten wurden nach l.c. (14), die Leukocyten nach l.c. (15) separicrt und durch mchrmaliges Einfrieren und Auftauen zerstört. Wir zentrifugierten $20 \mathrm{Min}$. bei $20000 \mathrm{~g}$. Der klare Uberstand wurde zur weiteren Untersuchung benützt. Die Erythrocytenmasse wurde in $\mathrm{NaCl}-L o ̈ s u n g ~ s u s p e n d i e r t ~ u n d ~ z e n t r i f u g i c r t$, was $8 \mathrm{mal}$ wicderholt wurde. Dic gewaschenen Erythrocyten wurden im $10 \mathrm{fach}$ em Volumen $1 \mathrm{~mm}$ Trispuffer $\mathrm{pH} 7$ hämolysicrt und $30 \mathrm{Min}$. bei $20000 \mathrm{~g}$ zentrifugiert. Zur Entfernung des Hämoglobins wurden die Nichthämoglobin-Eiweiße des Hämolysats an DEAE-Cellulose adsorbiert. Diese wurde mit $1 \mathrm{~mm}$ Trispuffer pH 7 bis zur Entfernung des Hämoglobins gewaschen. Die auf DEAE-Cellulose adsorbierten Erythrocyteneiweiße wurden mit $0,5 \mathrm{M} \mathrm{NaCl}$ eluiert und "mittels Ultrafiltration konzentriert. Im Untersuchungsgut wurde das Gesamt-Eiweiß durch die Biuretmethode nach SoLs (16) und die Gesamt-Aldolaseaktivität nach KuLganeK und KLASCHKA (17) bestimmt. Danach wurden dis Zellprotcine der Elektrophoresc unterzogen, um dic Isoenzyme der Aldolase zu trennen. Die Elektrophorese wurdc in 0,6proz. Agarosegcl in Plexiglaströgen $(180 / 130 / 4 \mathrm{~mm})$ durchgeführt, wobei Filterpapierstreifen als Kontakt zwischen Gel und Puffer in den Küvetten dienten. Der Puffer bestand aus Tris/ $\mathrm{H}_{3} \mathrm{BO}_{3} /$ EDTA nach Aronson und Grönwall (18). Die Trennung dauerte $18 \mathrm{Stdn}$. bei $160 \mathrm{~V}$ und $35 \mathrm{~mA}$ und $2^{\circ}$. Die Lokalisierung der Aldolascaktivität wurde nach demselben Verfahren, wic in 1. c. (13) durchgeführt.

\section{Ergebnisse und Diskussion}

Die Ergebnisse über die Gesamtaktivität der Blutzellenaldolase sind in Tabelle 1 dargestellt.

Hieraus ist $\mathrm{zu}$ ersehen, daß die Leukocyten die größte Gesamtaldolaseaktivität besitzen. Gegenüber LöHR

Tab. 1

Spezifische Gesamt-Aldolaseaktivität der Blutzellen (U/ing Zellprotein, $3^{\circ}$ ). Substrat: Fructose-1,6-diphosphat. $\overline{\mathbf{x}}$ - Mittelwert; $\mathbf{s}=$ Standardabweichung

\begin{tabular}{cccc}
\hline & Erythrocyten & Leukocyten & Throinbocyten \\
\hline$\overline{\mathbf{s}}$ & $\pm 0,57$ & 1,60 & 0,27 \\
$\mathbf{S}$ &, \pm 17 & $\pm 0,29$ & $\pm 0,073$ \\
\hline
\end{tabular}


und Mitarbeitern (10) finden wir in den Erythrocyten eine höhere Aldolaseaktivität als in den Thrombocyten. Dies ist darauf zurückzuführen, daß diese Verfasser die Aldolaseaktivität in vollständigem Hämolysat bestimmen und auf Gramm Hämoglobin beziehen. Wir dagegen bestimmen die Aldolaseaktivität nach Entfernung des Hämoglobins und beziehen sie auf Milligramm Nichthämoglobin-Erythrocyteneiweiß.

Bei der Untersuchung des Isoenzymmusters der Aldolase in den Blutzellen benützten wir als Substrat nicht nur das Fructose-1,6-diphosphat (Nachweis der Fructosediphosphat-Aldolase (EC 4.1.2.13)), sondern auch das Fructose-1-phosphat (Nachweis der Ketose1-phosphat-Aldolase (EC 4.1.2.7)). Auf Grund des von uns vorgeschlagenen Verfahrens (13) ist die Anwendung von Fructose-1-phosphat als Substrat deshalb möglich, da das freigesetzte Glycerinaldehyd mit der der Inkubationlösung in hoher Konzentration zugefügten Glycerinaldehyd-3-phosphatdehydrogenase in Reaktion treten kann (19).

Bei Anwendung des Fructose-1,6-diphosphats als Substrat erhielten wir folgende Isoenzymmuster der Blutzellen. (Abb. 1). Bei den Erythrocyten (Abb. 1a und a') besteht das Isoenzymmuster aus 2-3 Fraktionen, die nahe bei der Startlinie an der anodischen Seite liegen. Sie sind vom Typ der Muskel-,„A“-Aldolase und entsprechen ihrer Beweglichkeit nach den $x_{1}$ und $\mathrm{x}_{2}$ Eiweißfraktionen der Erythrocyten, wie in einer früheren Arbeit beschrieben (20, 21). Außerdem

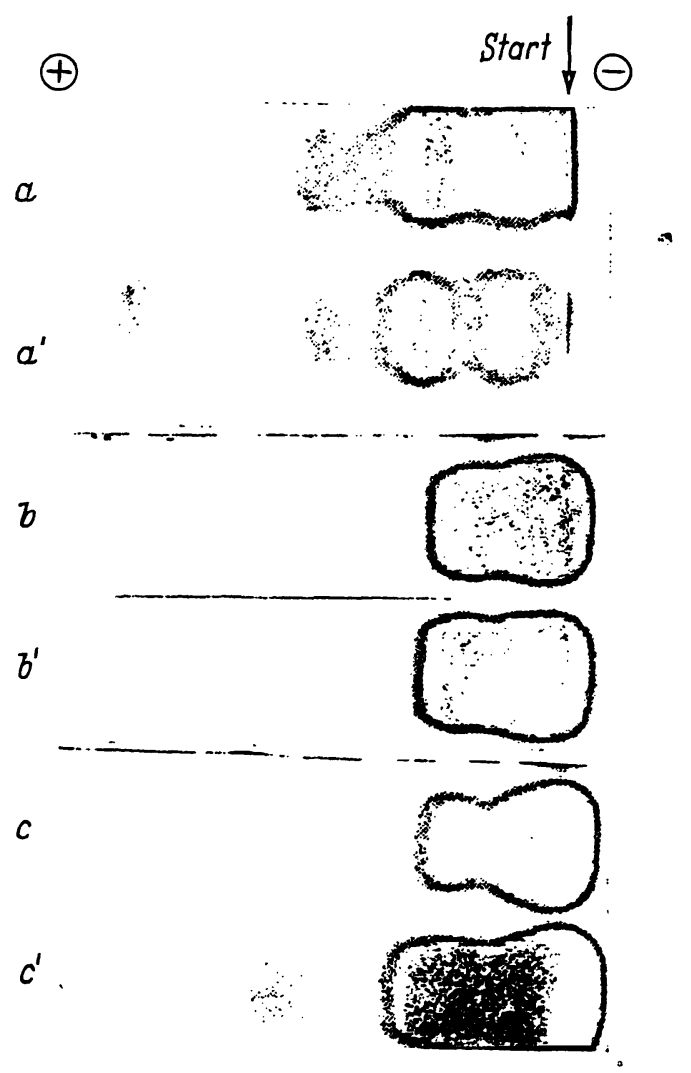

Abb. 1

Isoenzymfraktionen der Blutzellenaldolase gesunder Menschen. Substrat Fructose-1,6-diphosphat

a und $a^{\prime}$ in Erythrocyten; $b$ und $b^{\prime}$ in Leukocyten; $c$ und $c^{\prime}$ in Thrombocyten werden noch 3 Isoenzymfraktionen gefunden, die noch näher an der anodischen Seite liegen. Die letzte von ihnen ist ziemlich intensiv. Sie entsprechen den "c" „d"- und „,"“-Eiweißfraktionen der Erythrocyten (20, 21) und sind vom Typ der Gehirn-,,C"-Aldolase.

Im Isoenzymmuster. der Leukocyten (Abb. $1 \mathrm{~b}$ und $\mathrm{b}^{\prime}$ ) finden wir eine Isoenzymfraktion an der Startlinie und nahe bei ihr, an anodischer Seite, noch drei Fraktionen. Dieser vier Isoenzymfraktionen sind vom Typ der

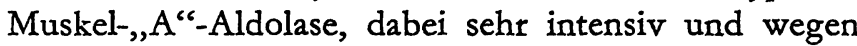
ihrer nahen Lage laufen sie fast ineinander. Anodisch von ihnen liegen noch 2-3 Fraktionen mit sehr schwacher Intensität vom Typ der Gehirn-,,C“-Aldolase.

Das Isoenzymmuster der Thrombocytenaldolase (Abb. $1 c$ und $\left.c^{\prime}\right)$ besitzt ebenfalls eine sehr intensive Fraktion, die an der Startlinie liegt und drei anodisch von ihr liegende Fraktionen vom Typ der „A“-Aldolase. Gegenüber den Leukocytenenzymfraktionen sind diese besser voneinander getrennt. Anodisch gelegen, finden wir noch drei Isoenzymfraktionen mit schwacher Intensität vom Typ der Aldolase-, ,C“.

Auf Abbildung 2 sind die Isoenzymmuster der Blutzellenaldolase unter Anwendung von Fructose-1-phosphat als Substrat veranschaulicht. Im Isoenzymmuster der Erythrocyten (Abb. 2a), der Leukocyten (Abb. 2b) und der Thrombocyten (Abb. 2c) erschienen nur die Isoenzymfraktionen der Aldolase- „C“. Dies stimmt mit den Untersuchungen einiger Verfasser (22) überein, $\mathrm{da} B$ die Gehirn-„C"-Aldolase hinsichtlich ihrer Spezifität gegenüber den Fructosephosphaten eine Mittelstellung zwischen den Typen „A“- und „B“-Aldolase einnimmt. Die Isoenzymfraktionen der Aldolase, die sich nahe und auf der Startlinie an der anodischen Seite befinden, traten nicht auf, da sie vom Typ der Aldolase- „A“" sind, die eine sehr niedrige Aktivität gegenüber Fructose-1-phosphat aufweist.

Wegen ihrer niedrigen Aktivität gegenüber Fructose-1phosphat wird die Erythrocytenaldolase von einigen Verfassern als reiner Typ „A“ bezeichnet (23). Die

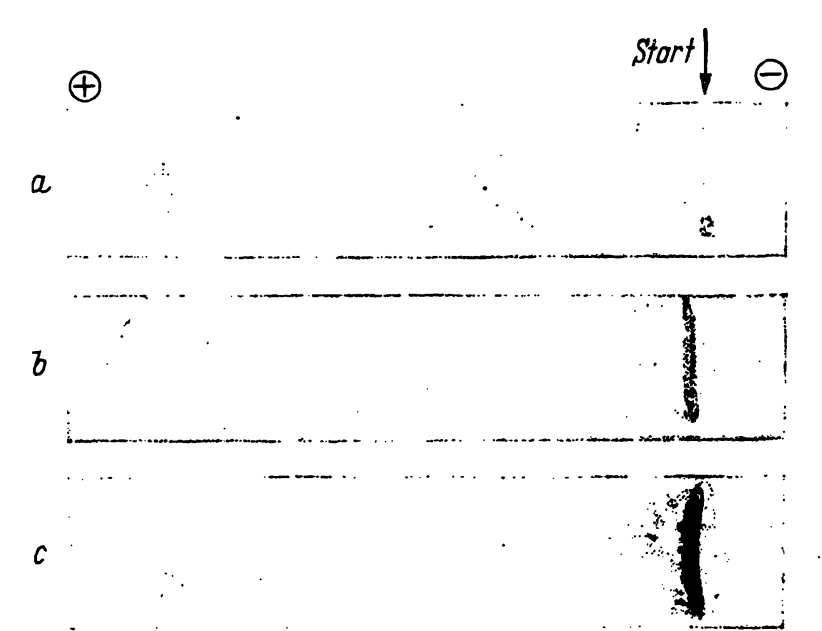

Abb. 2 Isoenzymfraktionen der Blutzellenaldolase gesunder Menschen.

a in Erythrocyten; b bei Leukocyten; c bei Thrombocyten 
Anwendung beider Substrate zum Nachweis von Isoenzymfraktionen ließ uns feststellen, daß die Blutzellen auch Isoenzymfraktionen vom Typ Aldolase „C C besitzen. Demnach können die Isoenzymfraktionen der Aldolase nicht nur nach der elektrophoretischen
Beweglichkeit bestimmt werden, sondern auch nach ihrer Substratspezifität. Daraus kann man schließen, zu welchem Aldolasentyp sie gehören.

Wir danken der Fa. Boehringer Mannheim für dic freundliche Uberlassung von Fructose-1-Phosphat.

\section{Literatur}

1. Löhr, G. W. und H. D. WAller, Klin. Wschr. 37, 833 (1959). - 2. Bruns, F., Biochem. Z. 325, 429 (1954). - 3. LöHR, G. W. und H. D. WAlleR, Dtsch. med. Wschr., 86, 27 (1961). - 4. AltManN, K. I., Amer. J. Med., 27, 936 (1959). - 5. Fornani, G., Giorn. biochem. 13, 420 (1964). - 6. Temkine, H., Bull. Soc. chim. biol., Paris 48, 771 (1966). - 7. LöHR, G. W. und H. D. WAlleR, Dtsch. med. Wschr. 89, 171 (1964). - 8. BECK, W. S., J. biol. Chemistri 216, 33 (1955). - 9. Vertrer, K., Fol. haemat., N. F. 6, 80 (1961). - 10. LöI1R, G. W., H. D. WALLER und R. Gross, Dtsch. med. Wschr. 86,897 (1961). - 11. Schetrini, F., M. B. Canani und F. Di Francesco, Boll. Soc. ital. biol. sper. 37, 1015 (1961). - 12. Dastugue, G., P. Bastide und P. M. Plat, Nouv. Rev. Franc. Hémat. 6, 265 (1966). - 13. Dikow, A. L. dicse Z. 6, 386 (1968). - 14. DuCkert, F., P. FlüCKIGer, H.
Isenschimid, M. Matter, J. Vogei-Menc und F. Koller, Acta haemat. 12, 197 (1954). - 15. WrLManNs, W., dicse Z. 5, 233, (1967). - 16. Sors, A. Nature London 160, 89, (1947). - 17. Kulganek, W. und W. Klaschika, Vopr. med. chimii (UdSSR) 7, 434 (1961). - 18. Aronson, T. und A. Grönwall, Scand. J. Clin. Invest. 9, 338 (1957). - 19. Harting, J. und S. F. Velick, J. biol. Chemistry 207, 857 (1954). - 20. 'ToDorow, J. und A. I. Drkow, Protides of the Biol. Fluid, Proc. 10th Collog. Brugges 1962 (ed. H. Peeters) Elsevier, Amsterdam (1963). - 21. ToDoRow, J. und A. L. Drkow, Protides of the Biolog. Fluides, Proc. 11th Colloq. Brugges 1963 (ed. H. Peeters) Elscvier Amstcrdam (1964). - 22. Penhoet, E., T. Rajkumar und W. J. Ruțer, Proc. nat. Acad. Sci. USA 56, 1275 (1966). - 23. PokrowskII, A. A. und I. I. Mussis, Biochimia (UdSSR) 31, 1197 (1966).
Dr. A. J. Dikow Bul. Chr. Botew 14 Sofia/Bulgarien 